\title{
EFFECT OF INTERSTRAIN PREGNANCY ON THE IMMUNE STATUS OF FEMALE MICE SENSITIZED TO PATERNAL ANTIGENS
}

\author{
G. A. GURRIE* \\ Edgar Laboratories, Fulham Hospital, London
}

(Received 24th June 1970)

The factors responsible for the immunological autonomy of the mammalian conceptus are poorly understood (Billingham, 1964). Pregnancy is unaffected by a variety of immunological insults. Immunization of the female against allogeneic paternal tissue has no effect on subsequent pregnancies (Lanman, Dinerstein \& Fikrig, 1962). Previous studies (Currie, 1969) have confirmed the observations of Breyere \& Barrett (1960) that pregnancy in the mouse induces a specific immunological defect involving paternal strain transplantation antigens and have extended them to show that this non-reactivity may be a form of antibody-mediated immunological enhancement. It is possible that this nonreactivity may play a part in protecting the conceptus from immune destruction.

This communication describes a simple experiment designed to assess the effects of inter-strain murine pregnancy across a major histocompatability barrier on the immune status of females previously immunized against paternal tissue and to show whether pregnancy-induced non-reactivity can overcome specific immunity. Sixty virgin mice were used in this experiment and were 8-week-old females of $A_{2} G$ strain and highly inbred. Groups of these females were given intraperitoneal injections of CBA-strain male spleen cells, each mouse receiving $6 \times 10^{7}$ viable nucleated cells in $0.2 \mathrm{ml}$ tissue culture medium 199. Control groups of females were given TC 199 only. Ten immunized and ten control females were not mated. The remaining immunized $\mathrm{A}_{2} \mathrm{G}$ females were mated 4 days later with male mice of proven fertility over a period of 5 days. One group of twenty females was mated with CBA-strain males and another similar group mated with $A_{2} G$ males. All the females were then weighed daily and only those with typical pregnancy weight gains 14 days after the start of the mating were used in the experiment.

The male strain test graft was a CBA-strain-specific, transplantable fibrosarcoma. It is readily rejected in a predictable fashion by $100 \%$ of all $\mathrm{A}_{2} \mathrm{G}$ females inoculated. Four groups of ten $\mathrm{A}_{2} \mathrm{G}$ females were injected subcutaneously in the shaved right flank with $0.2 \mathrm{ml}$ of a $50 \%$ tumour mince in TC 199. The four groups injected were: non-immune non-pregnant $A_{2} G$, immune nonpregnant $A_{2} G$, immune 14-day pregnant by $A_{2} G$ males (intra-strain) and immune 14-day pregnant by CBA males (inter-strain). Maximum tumour

* Present address: Chester Beatty Research Institute, Institute of Cancer Research, Royal Cancer Hospital, Laboratories at Clifton Avenue, Belmont, Sutton, Surrey. 
diameters were measured daily using a transparent millimetre scale. The mean diameter growth curves for each group of mice are represented graphically in Text-fig. 1. Non-immune, non-pregnant, $A_{2} G$ females rejected the tumour within 10 to 14 days, during which time it grew to be about $1 \mathrm{~cm}$ in diameter before regression. Immune non-pregnant females rejected the tumour rapidly and only minute transient nodules were detectable. Pregnancy, whether intrastrain or inter-strain, had no effect on this rapid rejection of the paternal tumour by immunized mice. The pregnancies in all these females continued normally. Litter size, gestation length and foetal resorption rate were unaffected by pre-immunization.

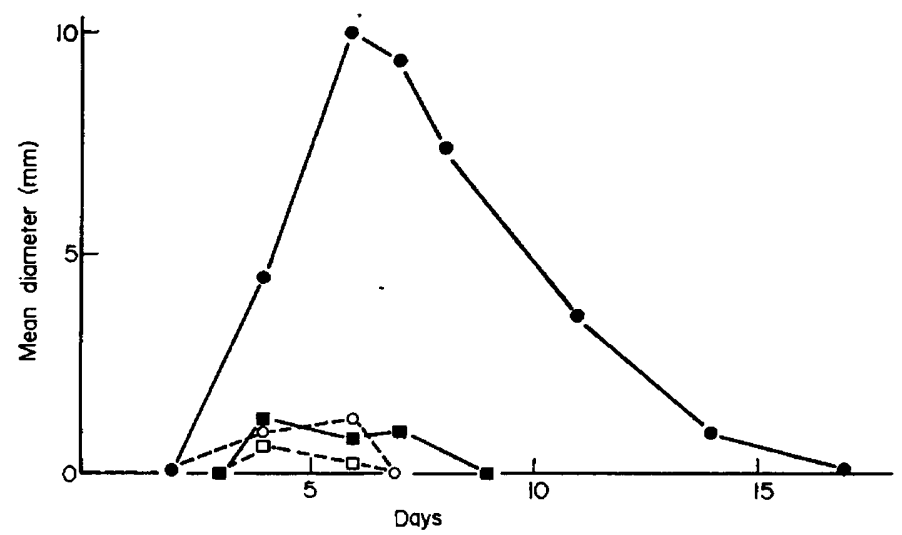

\begin{abstract}
TEXT-FIG. 1. Mean diameter growth curves of a CBA-strain sarcoma transplanted to $A_{2} G$-strain female mice. Pregnancy had no effect on the rapid rejection of this tumour induced by pre-sensitization. $\bullet$, Tumour growth in virgin $A_{2} G$ females; $O, A_{2} G$ virgin females sensitized to $\mathrm{CBA}$ antigens; $\boldsymbol{m}$, intra-strain pregnant $\mathrm{A}_{2} \mathrm{G}$ females sensitized to $C B A$ antigens; $\square$, inter-strain pregnant $A_{2} G$ females sensitized to $C B A$ (paternal) antigens.
\end{abstract}

This result indicates that established cell-mediated immunity to paternal tissues is detectable in pregnancy and is unaffected by it. In other words, any specific (or non-specific) non-reactivity to paternal antigens induced by pregnancy is too weak to have any effect on the immune status of pre-immunized females. Using histological examination of grafted tissues in rats and rabbits, Woodruff (1958) concluded that pregnancy did not affect the rejection of foetal limb fragments transplanted subcutaneously in females pre-sensitized by a paternal skingraft. This simple qualitative approach was not sensitive enough to detect any but the most dramatic changes in reactivity. Indeed, it failed to reveal any changes in reactivity in pregnant non-immunized females, a phenomenon readily demonstrable in mice using the growth of a tumour allograft as the test system (Currie, 1969).

That inter-strain pregnancy is unaffected by pre-immunization was also confirmed in this experiment, indicating that pregnancy-induced non-reactivity to paternal transplantation antigens, thought to be a form of immunological enhancement, has no important rôle in protecting the conceptus from immunological destruction. That such protection is the responsibility of a foeto- 
maternal barrier providing immunological isolation of the foetus (Currie, 1968) remains the most favoured hypothesis.

The author gratefully acknowledges a fellowship from the Wellcome Trust.

\section{REFERENCES}

Billingham, R. E. (1964) Transplantation immunity and the materno-foetal relation. New Engl. $\mathcal{f}$. Med. 270, 667.

BREYeRE, E. J. \& BARRETT, M. K. (1960) "Tolerance" in post-partum female mice induced by strain specific matings. F. natn. Cancer Inst. 24, 699.

Currie, G. A. (1968) The foeto-maternal barrier. Proc. R. Soc. Med. 61, 1206.

GURRIE, G. A. (1969) The foetus as an allograft: the role of maternal unresponsiveness to paternally derived foetal antigens. In: Foetal Autonomy. Giba Fdn Symp. Eds. G. E. W. Wolstenholme and M. O'Connor. Churchill, London.

Lanman, J. T., Dinerstein, J. \& Fikrig, S. (1962) Homograft immunity in pregnancy: lack of harm to the foetus from sensitization of the mother. Ann. N.r. Acad. Sci. 99, 706.

WOODRUFF, M. F. A. (1958) Transplantation immunity and the immunological problem of pregnancy. Proc. R. Soc. B, 148, 68. 